\title{
Using Innovation to Develop Digital Tools for Public Health During the COVID-19 Pandemic
}

\author{
Authors: \\ *Kate Mark,, Jay Bradley, ${ }^{2}$ Chaloner Chute, ${ }^{1}$ Colin Sumpter, ${ }^{3}$ Mahmood \\ Adil, ${ }^{3}$ George Crooks ${ }^{1}$ \\ 1. Digital Health and Care Innovation Centre, Glasgow, UK \\ 2. Glasgow School of Art, UK \\ 3. Public Health Scotland, Edinburgh, UK \\ *Correspondence to Kate.mark@dhi-scotland.com \\ Disclosure: $\quad$ The authors have declared no conflicts of interest. \\ Acknowledgements: The authors would like to thank all those who participated in the workshops, and \\ colleagues at StormID and NHS National Services Scotland. \\ Received: $\quad 17.09 .20$ \\ Accepted: $\quad 03.06 .21$ \\ Keywords: COVID-19, digital, innovation, pandemic, public health. \\ Citation: $\quad$ EMJ. 2021;6[3]:50-60
}

\begin{abstract}
Introduction: Technology has played a key role in enabling public health to respond to the COVID-19 pandemic at a pace and scale never seen before. The Digital Health and Care Innovation Centre (DHI) assisted with development of two new digital services to enable testing and contact tracing at scale using innovative methods.
\end{abstract}

Methods: The DHI employed a design innovation approach by bringing all relevant stakeholders together to co-design new technology services to identify the 'preferred future'. Workshops were used to identify the preferred solutions. The innovative methods for development of digital health tools included adopting an iterative approach, addressing the situational requirements posed by COVID-19, and democratising technology for purposes of pandemic control.

Results: A National Notification Service (NNS) for automation of delivery and feedback (if results messages were viewed) was developed and adopted by five of the 14 health boards in Scotland, processing over 7 million results since inception.

The Simple Tracing Tools (STT) is an open-platform web-based app that is designed for data entry by contact tracing teams. STT was adopted by all local health protection teams and informed development of the national case management system.

Discussion: The Cynefin framework can be used to understand the design innovation process when facing the challenges of designing digital tools during a pandemic. There are significant opportunities for public health to engage with digital health to transform the pandemic response and derive benefit for tackling future population health challenges. 


\section{INTRODUCTION}

This paper describes a case study of the co-design and development of two new COVID-19 digital services to support the Scottish Government's Test and Protect strategy.

All aspects of health services have adapted in response to the COVID-19 pandemic. Public health is at the forefront of the COVID-19 response and is no exception in embracing innovative ways of working. Technology has played a vital role in enabling public health services to manage clusters of COVID-19 as the scale and intensity of the pandemic threatened to overwhelm normal practice. ${ }^{2}$ Examples of innovative practice include developing novel digital technology to abet symptom checking, ${ }^{2}$ contact tracing, ${ }^{3}$ and quarantine compliance. ${ }^{4}$ In Scotland, public health has relied on 'legacy technology' and manual processes to carry out outbreak control. Expertise was not lacking, but the scale of the pandemic meant the service was under-resourced and unable to increase the workforce rapidly to respond. The digital infrastructure in public health required transformation to facilitate an increase in testing and contact tracing capacity.

The Digital Health and Care Innovation Centre $(\mathrm{DHI})$ is one of the Scottish Government's innovation centres funded by the Scottish Funding Council, working with NHS Scotland's Health Boards and Scotland's local authorities. They have a remit to improve Scotland's health using digital technology and encouraging economic growth for Scotland. ${ }^{5}$ Public Health Scotland (PHS) engaged with the $\mathrm{DH}$ to develop new digital tools and services that would support local Health Boards and national agencies to identify and manage outbreaks of COVID-19.

\section{Contact Tracing in Scotland}

Contact tracing is a well-recognised practice in public health that identifies individuals who may have been exposed to an infectious disease and providing them with advice to limit spread of the disease. In the case of COVID-19 in Scotland, that advice was to self-isolate for 10 days. ${ }^{6}$ Contact tracing in Scotland is led by the local health board Health Protection teams, with support from the national agency, PHS, if there are cross-border or international issues. National guidance and policies are adapted by boards to suit local practice and the available resources, with the result that contact tracing is similar from a strategic perspective across Scotland but may differ operationally with different data recording systems in place. Disease vector control often requires requesting or enforcing individuals to remove themselves from elements of society to protect others, while not necessarily benefiting themselves directly. ${ }^{7}$ This has borne the authoritative stance of Health Protection for certain actions such as tracing and quarantine; however, COVID-19 has illuminated the role of active participation by the citizen. ${ }^{8}$ Moving the citizen towards a central role in public health services is a paradigm shift from traditional health protection models, which adopts a more directive, service-led approach.

\section{Aim}

The DHI, in partnership with PHS and the National Health Service (NHS) National Services Scotland (NSS), were tasked with using their experience in innovation and digital health to develop digital services to assist the Scottish Government's Test and Protect strategy. ${ }^{9}$ Here, the authors describe the development process of two services that were co-designed over ten weeks between March 2020 and May 2020, using innovative methods:

1. The National Notification Service (NNS) is a service that automates the delivery of test results for COVID-19 across Scotland.

2. The Simple Tracing Tools (STT) is a web-based service to enable large numbers of individuals to carry out contact tracing at a local and national scale.

This paper will describe the new services and outline the process and innovation methods used by the $\mathrm{DHI}$, both in the design-led approach and the technological approach.

\section{The Challenge}

Two aspects of the 'test, trace, isolate, support' strategy ${ }^{10}$ were identified by public health specialists that could benefit from digital development in the immediate- to short-term: test result management and contact tracing. As contact tracing is usually initiated on a positive test result confirming a case, services were developed as separate but interdependent digital tools. 
The DHI formed a core team consisting of the DHI's Chief Technology Officer, a Design Researcher, a Public Health Innovation Fellow, and Public Health Specialist at PHS. This team organised and facilitated co-design workshops, analysed workshop outcomes, identified the expert participants required, and progressed the development of the tools through to the point of handover to NSS.

\section{METHODS}

The DHI held a series of 29 workshops over 92 days between $9^{\text {th }}$ March (first workshop) and $8^{\text {th }}$ June (final delivery) to co-design and deliver the two new services. This work began with an initial face-to-face design workshop held at Health Protection Scotland (HPS), the national health protection organisation, on $9^{\text {th }}$ March 2020. Participants at the workshops included public health experts, e-health leads, laboratory leads, and industry partners. The DHI and HPS invited the participants and some invitees elected to invite others whom they thought relevant. The $\mathrm{DHI}$ arranged this workshop at short notice in response to the developing COVID-19 situation in Scotland. After the initial workshop (the only face-to-face contact for this work), all following workshops and meetings were held using an online, virtual whiteboard to visualise concepts and online meeting services to facilitate conversation. The visual output from the analysis of each workshop was shared as widely as possible between workshops to help communicate new learning across a wide range of stakeholders (Figures 1 and 2). Workshops followed a participatory design approach. The $\mathrm{DHI}$ prepared visual scaffolding before each workshop to facilitate discussion between disparate stakeholders and ensure key challenges were addressed. Scaffolding highlighted the citizen as the centre of the service.

Participatory design was a useful methodology to support meaningful contributions from a wide range of participants, including public health experts, novice contact tracers, e-health leads, occupational health leads, sexual health leads, software developers, and information and clinical governance leads. Time pressures dictated that the partnership was not able to involve citizens in the co-design of these systems.
Workshops could be single discipline, or multidisciplinary across the fields, dependent on the questions and challenges unearthed. The DHI design researcher facilitated the workshops. The topics of each workshop would depend on the immediate requirements to inform progress, and included, in order:

\section{Which services are needed?}

\section{Service design model}

3. Prioritisation of features

4. Iterative design of prototypes

5. Future of service

6. Governance required to continue development

Topics were revisited with different experts and reiterated during the development of the two services. An iterative series of workshops informed the ongoing design. The software development team could also pose questions back to relevant experts to inform development through these workshops. To the best of the authors' knowledge, national public health services have not been co-designed by participants from across the health and technology professions. Specifically, not in response to a national pandemic.

\section{Service Needs Identified}

Participants co-produced the requirements for the services after agreeing on the services needed. Note that development started while the design of the services was still happening due to the speed at which the services needed to be delivered (Figure 3). For the citizen, the services needed to be accessible, respect privacy, acceptable, easy to use, improve the experience of the service, and promote self-management. For public health, the services needed to be effective, evidence-based, safe and secure, rapid, adaptable, reliable, not widen inequalities, and promote self-management.

\section{The Design Approach}

The DHI employs a design innovation approach, bringing relevant stakeholders together through participatory, design-led workshops to co-design new technology services." 

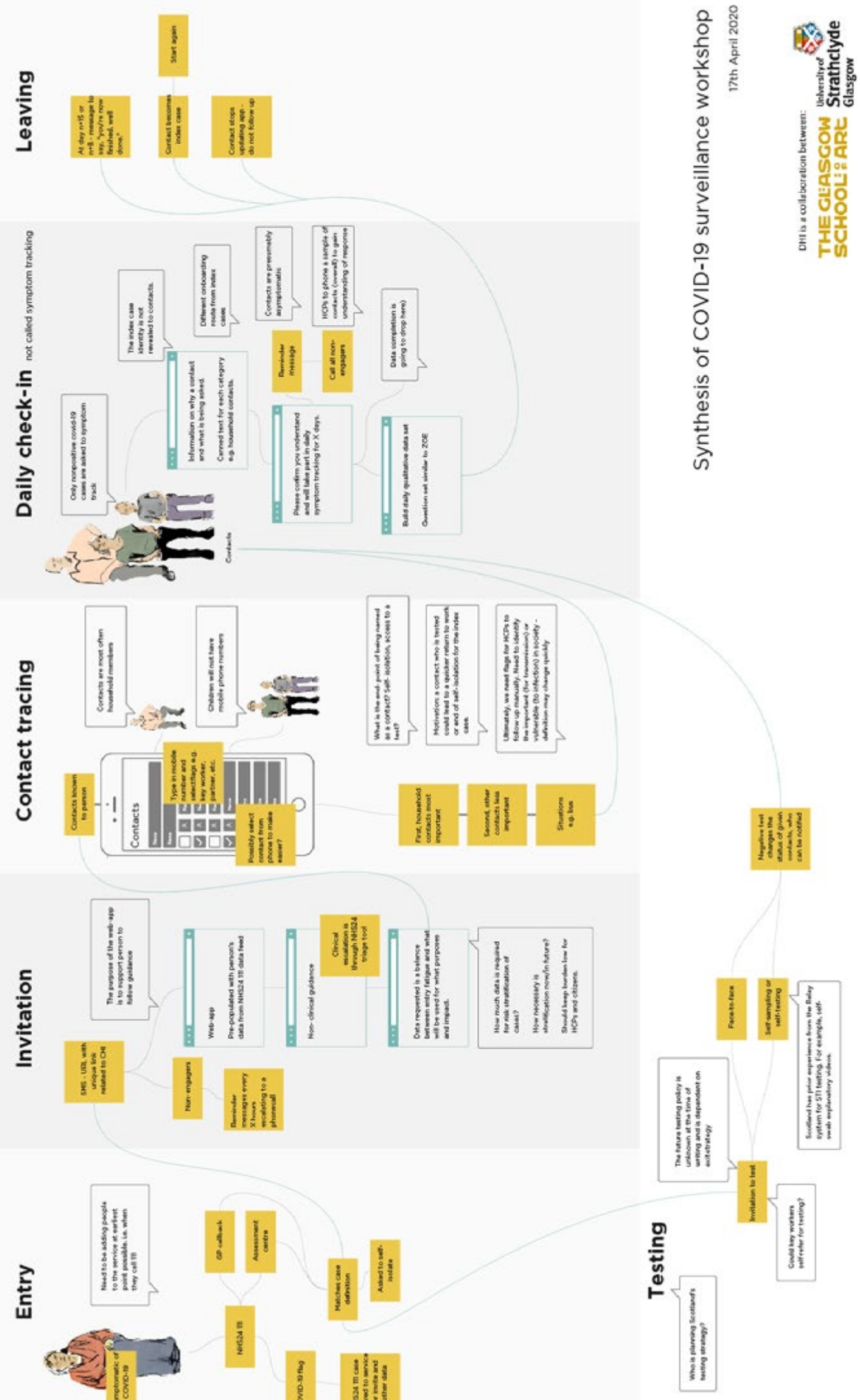

Figure 1: Possible future patient journey of 'testing, tracing, and isolating' developed during a remote co-design workshop when some aspects of what would become the Scottish Government's 'test, trace, isolate, support' approach were unknown.

The figure is indicative of the types of visual communication produced by participants during workshops. 


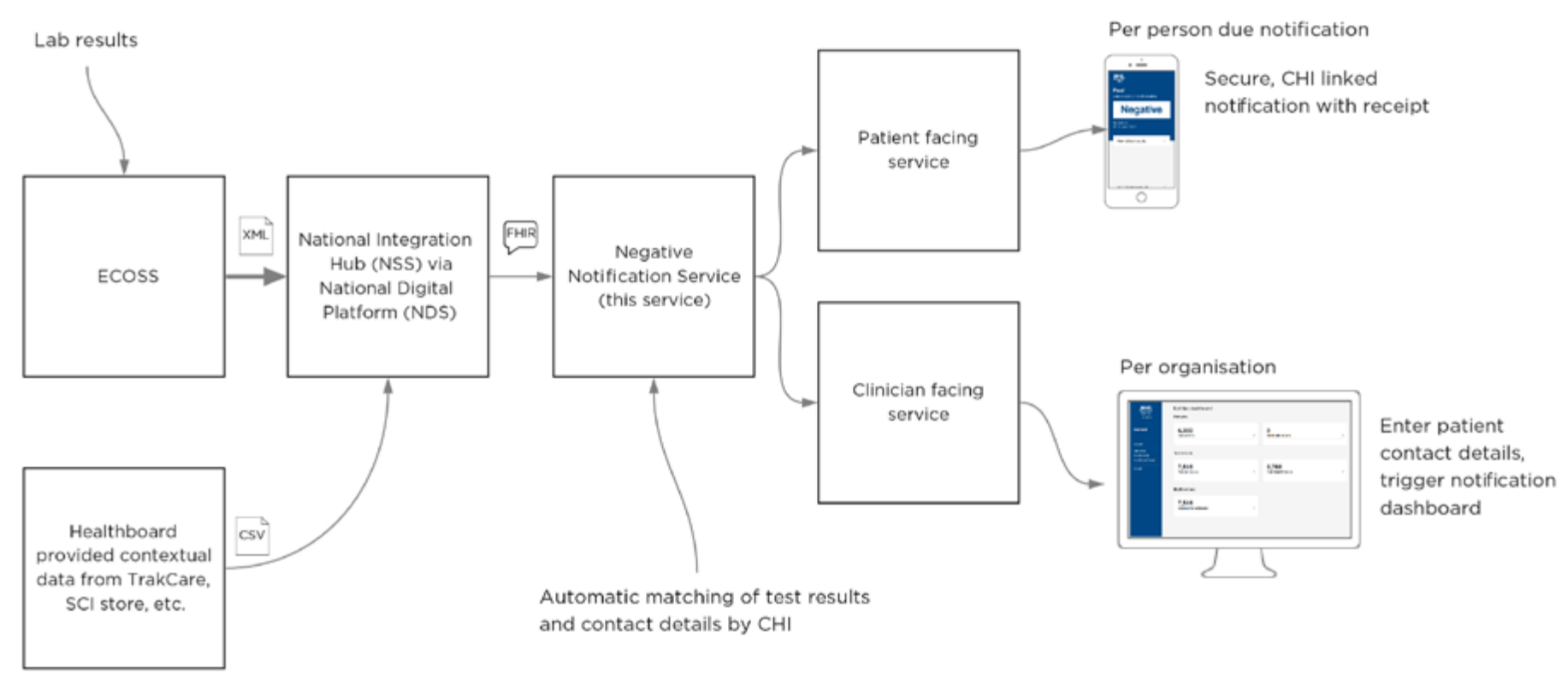

Figure 2: A co-designed, simplified diagram of data flow for the National Notification Service. Note that the Negative Notification Service became the National Notification Service during the project.

CHI: Community Health Index; CSV: comma-separated values; ECOSS: Electronic Communication of Surveillance in Scotland; FHIR: Fast Healthcare Interoperability Resources; NDS: NES Digital Service; NSS: Negative Notification Service; SCl: Scottish Care Information; XML: eXtensible mark-up language.

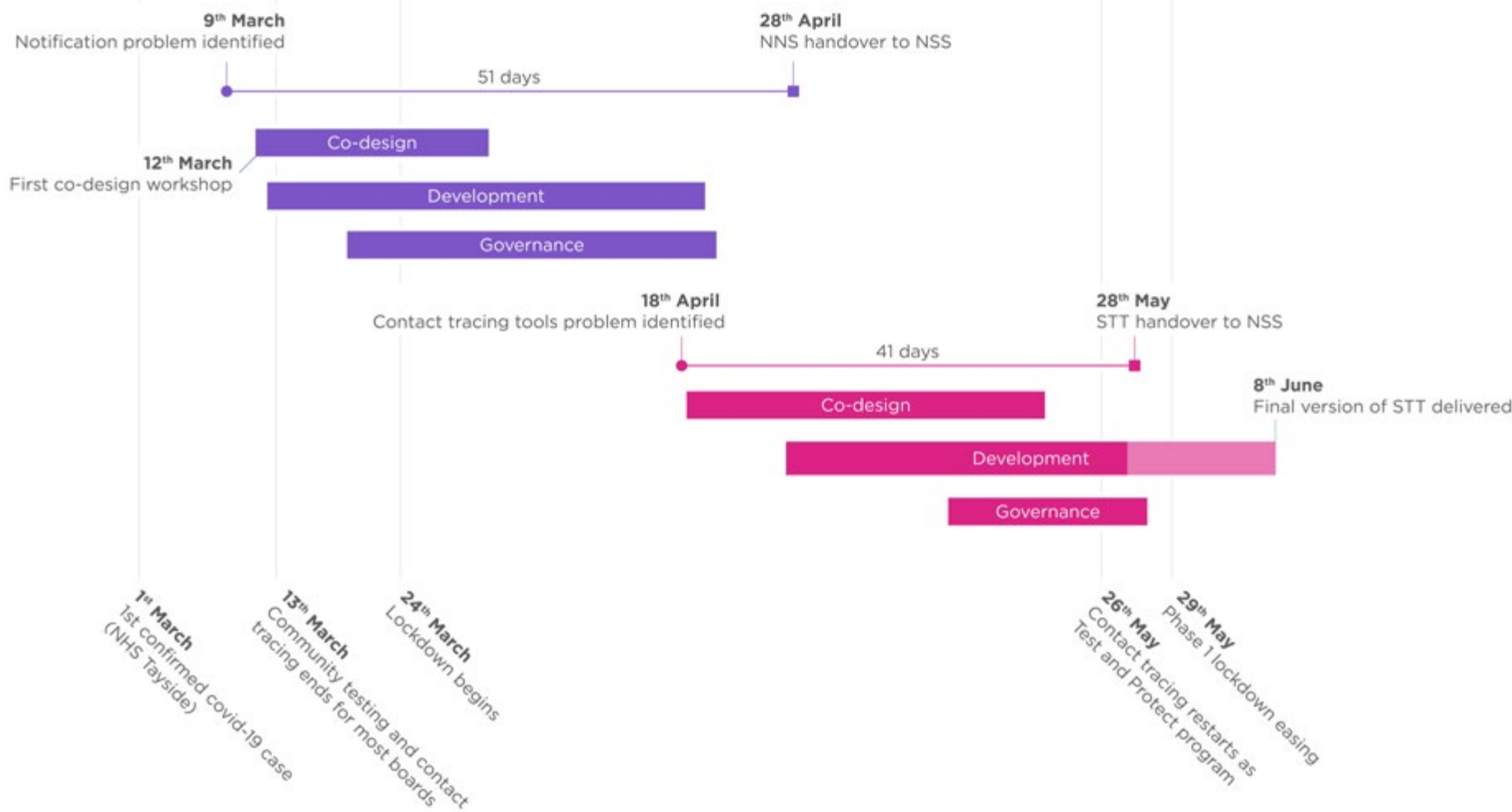

Figure 3: Showing the project timeline alongside significant pandemic dates.

NNS: National Notification Service; NSS: National Services Scotland; STT: Simple Tracing Tools. 
Table 1: Summary of features of Simple Tracing Tools and National Notification Service and relevance to service.

\begin{tabular}{|l|l|l|}
\hline & Patient story & Service story \\
\hline SMS to inform patient result available. & $\begin{array}{l}\text { I want to be able to receive my result } \\
\text { in a format that is fast and convenient. }\end{array}$ & $\begin{array}{l}\text { I want to be able to automate results } \\
\text { to ensure rapid dissemination. }\end{array}$ \\
\hline Unique URL to view a test result. & $\begin{array}{l}\text { I want to be the only person who can } \\
\text { see my result. }\end{array}$ & $\begin{array}{l}\text { I want to ensure the correct individual } \\
\text { receives the result. }\end{array}$ \\
\hline Feedback to service if result viewed. & $\begin{array}{l}\text { I want to know the service has } \\
\text { followed up my result. }\end{array}$ & $\begin{array}{l}\text { I want to know if the individual has } \\
\text { viewed their result as further action } \\
\text { may be required. }\end{array}$ \\
\hline $\begin{array}{l}\text { Restrict notification for some patient } \\
\text { cohorts, e.g., care home residents. }\end{array}$ & $\begin{array}{l}\text { I do not want to be sent my test result } \\
\text { multiple times. }\end{array}$ & $\begin{array}{l}\text { I do not want vulnerable patients or } \\
\text { families to receive results via SMS } \\
\text { inappropriately. }\end{array}$ \\
\hline
\end{tabular}

SMS: short message service; URL: uniform resource locator.

Ideally, digital services should meaningfully involve those that will use the service (e.g., healthcare staff) and those that will be subject to the future service (e.g., Scotland's citizens) in the design. In this way, those people's lived experience should be reflected in the delivered services and mistakes due to assumptions, or unknown problems and opportunities, should be minimised. The DHI call such a solution the 'preferred future', a future service that accounts for constraints but is the most preferable according to the appropriate people. As already noted, citizens were not included in this co-design due to limited time. Ideally, those subject to the new service would be meaningfully included in the design of it. Analysis was done during workshops by consent (not by the authors in isolation) and followed an 'analysis-on-the-wall' approach. ${ }^{12}$ That is, participants agreed upon what they had proposed through consensus and diagrams summarising and communicating the decisions were produced on the fly during workshops or were prepared for the next workshop. For example, a mostly empty version of Figure 1 was used for the initial discussion of the 'test, trace, isolate, support' strategy (before it was named TTIS). The diagram in Figure 1 was built during the workshop by participants (with facilitation) and only minor adjustments (e.g., spelling mistakes, alignment) were made before dissemination to stakeholders. This is true of Figure 2, which was also built using an online collaborative whiteboard during the workshops, by the participants, with facilitation.

\section{Identifying the Preferred Solution}

During the development process, the partnership posed the question: "What if we put the citizen at the centre of pandemic control and enabled them to self-manage testing and contact tracing?"

Through participatory design workshops, the DHI considered the imminent potential future where every citizen is engaged in pandemic control. The $\mathrm{DHI}$ and partners developed digital tools in an innovative and agile way, while accepting some answers or solutions were not readily available. Through open exploration, they could consider and progress the potential options (an approach sometimes termed negative capability ${ }^{13}$ in design research). Due to the conditions of the pandemic and changing landscape of policy, guidelines, and services, knowledge of both the virus and the system aiming to control the virus was in constant evolution. Additionally, the scale of the pandemic could only be estimated from modelling, and so best- and worst-case scenarios were anticipated.

\section{The Technological Approach}

A vital part of any innovation methodology is to foster the environment for creativity and change. The $\mathrm{DHI}$ does this through a Demonstration and Simulation Environment (DSE). ${ }^{14,15}$ 
significant challenge faced by innovators when implementing a novel digital technology within the NHS is overcoming the perceived risks of change. The DSE is a 'safe space' that enables clinicians, patients, and technologists to work together to redesign their underlying service delivery models with digital tools developed in tandem. The DSE produces simulated services that show how future, preferred services would be made without the constraints of existing health and care system constraints.

This collaborative 'de-risking' model focuses on the principles to 'learn by doing' and 'show not tell'. For COVID-19, the clinical users were part of developing the tools through concept co-design, into a simulated environment and then into real use as part of their live systems. Rapid iteration of features was possible to inform agile releases and collaboration with partners to develop the anticipated future state of a more extensive technical infrastructure and case management system. In this approach, Public Health agencies could develop their preferred possible contact tracing service. In this instance the services began as simulated services before being commissioned for deployment.

\section{RESULTS}

Through the design innovation processes, the DHI identified two significant aspects of pandemic control that would be improved through the co-design and adoption of novel digital tools in Scotland. Through collaborative workshops with relevant organisations, these two digital tools were co-designed, developed, and implemented within 10 weeks from conception to enable automated test notification and contact tracing at scale, as shown in Figure 3.

\section{Operational and Situational Technology Requirements Related to the COVID-19 Pandemic}

A solution that required minimal data integration was desirable. At the first workshop, the participants identified a single national source of results of COVID-19, using Electronic Communication of Surveillance in Scotland (ECOSS). At later workshops, the need to accept data feeds from the local health boards was identified. These two sources of data can be seen in Figure 2, which was used in some form in almost all workshops after being co-produced. Later again, additional test result feeds from new purpose-built lab systems (to enable local arrangements of services to be combined with national tools) were added.

Data collection and reporting is a crucial part of a pandemic response. The team built technological solutions using a whole-system data collection model that consistently applied a data dictionary derived from World Health Organization (WHO) guidance. ${ }^{16}$ This enabled the local health boards of NHS Scotland and PHS to collect data to inform epidemiological investigation.

The team worked with known industry software development partners, with trusted flexible capability from previous projects. ${ }^{15}$ Organisational trust in the system and risk management were prioritised throughout the development to ensure work could progress at pace. The tools were adapted using the available evidence base and learning from approaches in other countries and services. ${ }^{17,18}$ This approach balanced the challenge of integration and redevelopment of any external technology. It has also avoided a 'COVID-only' service, with the intention that the solutions would be a legacy for public health. Public trust in the Test and Protect system is essential for preventing the spread of COVID-19. As such, data collection was carefully considered by a range of experts to ensure only essential data was required. Data was not shared with any third-party, and all data was collected according to NHS data governance principles. A full Data Protection Impact Assessment (DPIA) was completed.

\section{Scaling the Technology}

The COVID-19 response necessitates services that can be scaled rapidly. Existing solutions had limitations in data sharing within and across organisations and remote working intensified these problems. The partnership's solution enabled a large number of contact tracers to carry out tracing without a virtual private network or additional equipment beyond a laptop. It allowed bespoke information governance processes to be applied to ensure appropriate governance for users without limiting the potential to scale and adapt to policy changes. The digital tools use an open data-exchange platform to allow for 
future extensibility of the service. Extensibility is particularly relevant considering the unknowns of COVID-19. Trusted third-parties can use an application programming interface to provide services such as information provision, selfserve contact tracing, and vaccine records. The nationally standardised services allow for variation in practice by different health boards in Scotland.

\section{National Notification Service}

The NNS was the first digital tool conceived at the initial workshop in March 2020. The NNS was developed in response to a public health professional's direct request to aid automation of result delivery from COVID-19 tests carried out by NHS Scotland. The NNS has been adopted nationally in Scotland; it has now been used to process over 7 million test results. It has enabled automation of other services such as a citizen selfservice contact tracing form, which has also been adopted nationally in Scotland and, at the time of writing, accounts for $64 \%$ of contact tracing data submissions.

\section{Simple Tracing Tools}

The STT were developed in recognition that to be able to contain the pandemic, contact tracing at scale was required..$^{19}$ In Scotland, contact tracing had been carried out using a proprietary Public Health system (HPZone $)^{20}$ or local methods using general-purpose tools such as Microsoft Excel $^{\circledR}$ (Microsoft, Redmond, Washington, USA). STT sought to understand the implications of a service change and ultimately informed the national case management system and service design of the National Contact Tracing Centre (NCTC). ${ }^{21}$ STT are built on an open dataexchange platform as web applications designed for flexible access and data entry by contact tracing teams. Following an initial seven-day pilot of three health boards, STT were adopted by all 14 local health protection teams to carry out contact tracing between $28^{\text {th }}$ May and $17^{\text {th }}$ July 2020. During this period, 733 professional users were onboarded, who traced 1,618 index cases, 2,273 contacts, and identified 605 settings.

\section{DISCUSSION}

\section{Collaboration}

The development and implementation of two digital services in 10 weeks, used by hundreds of public health professionals across Scotland, results from the success of the collaboration between agencies, including trust in each organisation's role and expertise. The $\mathrm{DHI}$ brought innovation, participatory design expertise, and considerable experience in technology infrastructure ${ }^{15}$ to NHS Scotland. PHS brought leadership, expertise, and high engagement levels with professional users (the local health boards who have responsibility for contact tracing within their geographical area). ${ }^{22}$ NHS NSS brought together the organisational threads required for implementation including information governance, clinical governance, and Equality Impact Assessments. ${ }^{23}$ The software development industry partners had experience working with the $\mathrm{DHI}$ and $\mathrm{NSS}^{15}$ and so the partnership could rely on their technical capacity and capability. The pandemic meant bureaucratic processes were expedited to ensure tools were deployed as soon as available, whilst participatory-design methods accelerated the design and implementation.

\section{Challenges of Innovation During a Pandemic}

As the time resource of experts was severely limited, efficient and informed decision-making was required. The pressure to resume contact tracing meant STT development had to pivot significantly from what was initially envisaged as a citizen-facing self-completion tool (which was later developed after the services described here) to a service-facing tool. All agencies accepted a riskier environment than normal practice would allow but recognised the opportunity to control specification through direct feedback into the development process during live use. In these conditions, the ability to simulate services in nonlive settings gave people more confidence to make decisions and enact development. Public health services were strained due to the ongoing COVID-19 response, ${ }^{24}$ and implementing a new service was a challenge due to the overburden of tasks. The innovation-adoption cycle ${ }^{25}$ was visible with variation in user engagement, with some 
individuals ('early adopters') highly motivated to design and adopt the service, while others were more reticent about a new digital product ('laggards'). There was a high risk of innovation overload and, although digital tools can reduce workload, there can be a significant burden in the implementation. ${ }^{26}$ It is a reminder that technology should not be forced onto a service that does not need it, or if its deployment creates more effort than it purports to reduce.

\section{Understanding the Design Process in Retrospect}

Here, the partnership used the Cynefin framework in retrospect to better understand the design and development process. ${ }^{27}$ It proposes that projects start from one of five domains: disorder, chaos, complex, complicated, and simple, and then move to a more well-understood domain in order. At the first workshop in March 2020, participants were in a disordered or chaotic situation with the worst-case pandemic scenario and relevant solutions both unknown. Tensions at this workshop were relatively high, and the opinion on the pandemic's scale and impact varied. Through participants' contributions at the first workshop, the stakeholder group moved from a chaotic to a complex problem domain. That is, some decisions were made, data sources were identified, and partners were either proposed or were in attendance and agreed to help. Through several more remotely run workshops, the partnership moved to the complicated domain, whereupon requirements and understanding settled. At that point, much more work still needed to be done but the design was mostly in place. At this point, expertise took over from design-led participatory design workshops. For example, industry partners began developing the service, and information governance documents were started. The stakeholders were then in the simple domain. A lot of work was still to be done, such as documentation for training, but it was only a matter of time and work before the services could be used.

\section{Public Health Implications}

This digital tool development experience has great implications for wider public health services, both in health protection and improving population health. The COVID-19 response requires a wholesystems approach with healthcare services, citizens, and political agencies collaborating and co-operating to prevent the spread of disease, ${ }^{28}$ and mitigating the harmful effects of necessary measures such as quarantine. ${ }^{29}$ Testing and contact tracing are key elements of this system, and digital tools can enable a whole-system approach to the containment of severe acute respiratory syndrome coronavirus 2 .

Throughout this process, the DHI used principles inherent to good practice in public health such as understanding the evidence base, considering and mitigating any adverse impacts on inequalities, and recognising the importance of successful engagement with the individual in population health policy.30 Other countries have adopted a more authoritarian approach to digital tools and contact tracing, ${ }^{31}$ which demonstrates the need to understand the broader political, cultural, and social forces in public health interventions. By understanding the importance of respect for privacy and responsible use of data, ${ }^{32}$ the partnership was quickly able to develop a societally acceptable service for test result delivery and contact tracing. The authors believe that the services are societally acceptable due to the consent of many healthcare professionals involved in their design, the inclusion of the DHI's proposed services in the Scottish Government's early TTIS strategy, ${ }^{10}$ and their mandated use across all 14 Scottish health boards by the Scottish Government.

COVID-19 has forced public health teams to act on a scale never seen before across the world. The pandemic's scale is a prime reason digital technology has been developed and deployed at an unprecedented pace. Digital technology can enable services to process and automate large numbers (whether it is test results, case management, or contact isolation support). ${ }^{33}$ These digital services can allow large amounts of data to be collected by the service to enable real-time epidemiology. ${ }^{3}$ Another considerable advantage of developing bespoke digital tools is that as collective understanding of COVID-19 has increased, they can be adapted to ensure it is capable of collecting the required data to inform risk assessment such as individual risk factors including ethnicity, and deprivation using the Scotland Index of Multiple Deprivation (SIMD), ${ }^{34}$ and accommodate complex settings that have emerged such as factories ${ }^{35}$ and activities. ${ }^{36}$ The stakeholders have been able to align the tool with 
changes in guidance and policy as the pandemic has progressed and lockdown eased.

\section{CONCLUSION}

The DHI, in partnership with PHS and NSS, rapidly developed and deployed two public health services in response to the pandemic, using a participatory design approach.

Digital tools should be adopted when they can enhance care, and service needs should dictate the nature of the tools (i.e., avoid digital for digital's sake). For example, the partnership chose not to develop proximity apps (as in the UK and beyond), instead focusing on a wholesystem digital approach as the preferrable solution decided at the workshops by relevant stakeholders.

Digital health is becoming an integral part of all aspects of healthcare including direct clinical care, self-management, and population health management. Public health should be fully engaged in digital healthcare, as there are considerable gains to be made. This engagement should extend beyond the COVID-19 pandemic. The lessons that could be learned from this global crisis of how digital tools can inform citizens, gather real-time data, and deliver services rapidly, and at scale. ${ }^{33}$ These lessons should apply to other public health interventions such as screening, ${ }^{37}$ vaccination programmes, ${ }^{38}$ and environmental exposure risk. ${ }^{39}$

The partnership's approach showed that digital health can have a fundamental role in public health services and can be developed at pace. To benefit from the legacy of the COVID-19 crisis, collaboration with relevant partners to inform the integration of ethical, equitable digital healthcare, and explore innovative ways of working together should continue.

\section{Footnote}

Health Protection Scotland (HPS) became part of the new organisation, PHS (Public Health Scotland) created on $1^{\text {st }}$ April 2020. The function of HPS remained unchanged throughout this period.

\section{References}

1. Whitelaw S et al. Applications of digital technology in COVID-19 pandemic planning and response. Lancet Digit Health. 2020;2(8):e43540 .

2. Menni $\mathrm{C}$ et al. Real-time tracking of self-reported symptoms to predict potential COVID-19. Nat Med. 2020;26(7):1037-40.

3. $\mathrm{Xu} \mathrm{B}$ et al. Epidemiological data from the COVID-19 outbreak, realtime case information. Sci Data. 2020;7(1):106.

4. Kleinman RA, Merkel C. Digital contact tracing for COVID-19. CMAJ. 2020;192(24):E653-6.

5. Digital Health and Care Institute. Available at: www.dhi-scotland.com. Last ac-cessed: 7 August 2020.

6. Public Health Scotland. COVID-19 contact tracing health protection team guidance version 3.4. 2020. Available at: https://hpspubsrepo. blob.core.windows.net/hps-website/ nss/3082/documents/1_covid-19- contact-tracing-guidance-hpt.pdf. Last accessed: 29 January 2021.

7. Magnusson R. "Controlling the spread of infectious diseases," World Health Organi-zation (eds.) Advancing the right to health: the vital role of law (2017) Geneva: World Health Organization, pp.151-64

8. Well, B. The "good" coronavirus citizen, the "covidiot", and the privilege of \#StayAtHome. 2020. Available at: https://discoversociety. org/2020/04/01/the-goodcoronavirus-citizen-the-covidiot-andthe-privilege-of-stayathome/. Last accessed: 13 August 2020.

9. Scottish Government. Coronavirus (COVID-19): Test and protect. Available at: https://www.gov.scot/ publications/coronavirus-covid-19test-and-protect/. Last accessed: 7 August 2020.

10. Scottish Government. Coronavirus (COVID-19): test, trace, isolate, support strategy. 4 May 2020. Available at: https://www.gov.scot/ publications/coronavirus-covid-19test-trace-isolate-support/. Last accessed: 13 August 2020.

11. Teal G, French T. Fast forward: accelerating innovation in health and wellbeing. 20 th $\mathrm{DMI}$ Academic Design Management Conference Inflection Point, 22-29 July, 2016.

12. Sanders EBN, Stappers PJ. Convivial Toolbox: Generative Research for the Front End of Design (2012) $12^{\text {th }}$ edition, Amsterdam: BIS Publishers.

13. McAra-McWilliam I. Impossible Things? Negative Capability and The Creative imag-ination. Creativity or Conformity, 8-10 January, 2007.

14. Chute, C.; Digital Health \& Care Institute. Introduction to the demonstration \& simulation environment (DSE). 2018. Available at: https://strathprints.strath. ac.uk/66279/. Last accessed: 12 August 2020.

15. Digital Health and Care Institute. $\mathrm{DHI}$ demonstration \& simulation 
environment: DHI phase $1 \mathrm{~b}$ report - lessons learned. 2019. Available at: https://strathprints.strath. ac.uk/68135/. Last accessed: 7 August 2020.

16. World Health Organization. Data dictionary for case-based reporting form. 2020. Available at: https://www. who.int/publications/m/item/datadictionary-for-case-based-reportingform. Last accessed: 12 August 2020.

17. Estcourt CS et al. The eSexual Health Clinic system for management, prevention, and control of sexually transmitted infections: exploratory studies in people testing for Chlamydia trachomatis. Lancet Public Health. 2017;2(4):e182-90.

18. Rimpiläinen S et al.; University of Strathclyde. Global examples of COVID-19 surveillance technologies: flash report. 2020. Available at: https://doi.org/10.17868/72028. Last accessed: 21 August 2020.

19. Royal Society DELVE Initiative. Test, trace, isolate. 2020. Available at: http://rs-delve.github.io/ reports/2020/05/27/test-traceisolate.html. Last accessed: 10 August 2020.

20. HPZone. InFact UK Ltd. 2016 Available at: https://hpzoneinfo.infact.com/. Last accessed: 10 August 2020.

21. Public Health Scotland. Scottish COVID-19 contact tracing programme. 2020. Available at: https://www.publichealthscotland. scot/our-areas-of-work/protectingour-health/scottish-covid-19-contacttracing-programme/. Last accessed: 10 August 2020.

22. NHS Scotland. Organisations. 2020. Available at: https://www.scot.nhs. uk/organisations/. Last accessed: 12
August 2020.

23. NHS Education for Scotland. Fairer Scotland and equality impact assessments. 2020. Available at: https://www.nes.scot.nhs.uk/ about-us/equality-and-diversity/ fairer-scotland-and-equality-impactassessments.aspx. Last accessed: 12 August 2020.

24. Pai M. Burnout, a silent crisis in global health. 20 July 2020 . Available at: https://www.forbes.com/sites/ madhukarpai/2020/07/20/burnouta-silent-crisis-in-global-health/. Last accessed: 12 August 2020.

25. Rogers EM et al. Complex adaptive systems and the diffusion of innovations. Innov J. 2005;10(3):29.

26. Buis L. Implementation: the next gian hurdle to clinical transformation with digital health. J Med Internet Res. 2019;21(11):e16259.

27. Snowden DJ, Boone ME. A leader's framework for decision making. Har Bus Rev. 2007;85(11):68-76,149.

28. Bradley DT et al. A systems approach to preventing and responding to COVID-19. EClinicalMedicine. 2020;21:100325

29. Thomas $\mathrm{J}$ et al.; University of Strathclyde. Global examples of methods and tools for supporting and incentivising self-isolation at home. 2020. Available at: https://doi. org/10.17868/72785. Last accessed: 10 August 2020

30. Satcher D, Higginbotham EJ. The public health approach to eliminating disparities in health. Am J Public Health. 2008;98(3):400-3.

31. Dubov A, Shoptawb S. The value and ethics of using technology to contain the COVID-19 epidemic. Am J Bioethics. 2020;20(7):W7-11.
32. Ienca M, Vayena E. On the responsible use of digital data to tackle the COVID-19 pandemic. Nature Med. 2020;26(4):463-4

33. eHealth Network. Mobile applications to support contact tracing in the EU's fight against COVID-19. 2020. Available at: https://ec.europa.eu/ health/sites/health/files/ehealth/ docs/covid-19_apps_en.pdf. Last accessed: 13 August 2020.

34. Scottish Government. Scottish Index of Multiple Deprivation 2020. 2020. Available at: https://www.gov. scot/collections/scottish-index-ofmultiple-deprivation-2020/. Last accessed: 12 August 2021

35. BBC News. Coronavirus: Chicken factory staff isolate after outbreak. 16 June 2020. Available at: https://www. bbc.co.uk/news/uk-wales-53062885. Last accessed: 10 August 2020.

36. Quinn A. COVID-19 Cluster in NI: Shared karaoke microphone infected up to $20 \mathrm{NI}$ party-goers with Coronavirus. 17 July 2020. Available at: https://www.newsletter.co.uk/ health/coronavirus/covid-19-clusterni-shared-karaoke-microphoneinfected-20-ni-party-goerscoronavirus-2917186. Last accessed: 10 August 2020

37. NHS Digital. Breast Screening Services. 2020. Available at: https:// digital.nhs.uk/services/screeningservices/breast-screening-services. Last accessed: 10 August 2020.

38. Wilson $\mathrm{K}$ et al. Travel vaccines enter the digital age: creating a virtual immunization record. Am J Trop Med Hyg. 2016;94(3):485-8.

39. Nyarku M et al. Mobile phones as monitors of personal exposure to air pollution: is this the future? PLOS ONE. 2018;13(2):e0193150 